\title{
Trois réécritures roumaines d'un voyage en Chine : celui de Nicolae Milescu
}

Hélène Lenz

\section{(2) OpenEdition}

1 Journals

\section{Édition électronique}

URL : https://journals.openedition.org/cher/2129

DOI : 10.4000/cher.2129

ISSN : 2803-5992

\section{Éditeur}

Presses universitaires de Strasbourg

\section{Édition imprimée}

Date de publication : 29 mai 2018

Pagination : 63-72

ISBN : 979-10-344-0019-5

ISSN : 1968-035X

\section{Référence électronique}

Hélène Lenz, «Trois réécritures roumaines d'un voyage en Chine : celui de Nicolae Milescu », reCHERches [En ligne], 20 | 2018, mis en ligne le 01 décembre 2021, consulté le 02 décembre 2021. URL : http://journals.openedition.org/cher/2129; DOI : https://doi.org/10.4000/cher.2129

\section{(c) (i) (2)(2)}

Ce(tte) œuvre est mise à disposition selon les termes de la Licence Creative Commons Attribution Pas d'Utilisation Commerciale - Partage dans les Mêmes Conditions 4.0 International. 


\title{
Trois réécritures roumaines d'un voyage en Chine: celui de Nicolae Milescu
}

\author{
HÉLÈNE LENZ ${ }^{1}$
}

$\mathrm{R}^{\mathrm{c}}$ écit de voyage rédigé en russe par un Moldave du XvII siècle en relations avec Louis XIV (Bărbulescu 1974: V), le Voyage en Chine de Nicolae Milescu a été découvert dans l'espace linguistique roumain par B.P. Hașdeu en 1866. S’il a été commenté en 1883 par une monographie d'Émile Picot, de l'École des Langues Orientales, il sera transposé dans la langue de sa culture d'origine pour la première fois en 1888 par Gheorghe Sion. Cette mise en forme moderne sera suivie au $\mathrm{xx}^{\mathrm{e}}$ siècle, à notre connaissance, d'une version de semi-vulgarisation d'E.M. Grigoraș (1926). L'édition en collection populaire de C. Bărbulescu (1974), parue à l'époque socialiste, alors que la dictature de Ceausescu se durcit, suite à un voyage du dirigeant roumain en 1971 dans la Chine de Mao-TséToung avec laquelle il établira des liens privilégiés - à l'instar de Jivkov, dictateur socialiste bulgare et d'Enver Hoxa, dictateur socialiste albanais - constitue le troisième avatar contemporain de cet écrit. Notre article consistera pour l'essentiel en un descriptif de ces trois réécritures, vues comme trois étapes idéologiques mais aussi scientifiques de la mise au jour d'un classique national.

Le mémoire français d'Émile Picot, postérieur à la création d'un premier État moderne roumain (1859), - composé des Principautés de Moldavie et Valachie -, suite à la rupture des relations avec la Sublime Porte en début de siècle, mentionne un "personnage extraordinaire» célèbre pour ses aventures et la diversité de ses «facultés».

Il appartient en même temps à l'histoire de la Moldavie, de la Grèce, de la Russie, de la Chine. Son origine, ses talents, ses crimes, la mutilation qu'il subit, le hardi voyage qu'il exécuta pour gagner Pékin en traversant toute l'Asie, les renseignements précieux qu’il rapporta de son ambassade auprès du Fils du Ciel,

1 Équipe d'accueil CHER, 4376, Université de Strasbourg. Toutes les traductions du roumain sont faites par Hélène Lenz. 
tout en lui concourt à exciter notre curiosité. Cependant l'histoire de Spatar ${ }^{2}$ est si peu connue que la plupart des orientalistes ignorent jusqu'à son nom de Milescu. Nous avons pensé que la Roumanie ne pouvait être mieux représentée dans un recueil consacré aux études orientales que par une notice [sur ses œuvres.] (Picot, 1883: 1)

En 2008, un symposium ${ }^{3}$ sur Milescu, ouvert par l'académicien Eugen Simion, annonçait une parution de ses écrits sous l'égide de l'Académie roumaine: il commémorait le troisième centenaire de la mort du lettré. Le Spathaire y a été montré comme une autorité savante du monde russe d'abord, européen ensuite. Ses connaissances linguistiques, incluant la maîtrise du slavon, langue liturgique chrétienne orthodoxe et idiome de la Chancellerie princière moldave du temps (Bărbulescu 1974: VIII, note1) lui ont permis d'obtenir la confiance du Métropolite de Constantinople avant celle du tsar qui l'a hébergé puis employé dans ses talents.

L'activité de lettré et diplomate de Nicolae Milescu atteint tout son éclat en Russie, où il arrive en janvier 1671, recommandé au tsar Alexei Mihalovic par le Métropolite Dosithée de Constantinople, en qualité de bon théologien et polyglotte. À la cour du tsar, le boïar moldave est reçu au Département des Ambassadeurs (Departamentul Solilor) comme interprète du grec, du latin, $\mathrm{du}$ roumain. Ses travaux en langue slavonne Aritmologion/Arithmologie et Cartea Sibilelor/Le livre des Sybilles datent de cette période. La renommée européenne de Nicolae Mlescu commence en 1675 quand il conduit une mission diplomatique en Chine. Un voyage difficile, aux péripéties nombreuses, fertile en écrits, qui lui vaudra l'appellatif de «Marco Polo de la Roumanie». Ce savant moldave est remarquable par son esprit d'observation aigu. Fin chercheur, il note pratiquement tout ce qu'il voit - de la configuration du sol à la vie des peuples, des hommes rencontrés avec leurs occupations, leurs coutumes, leurs armes, leurs manifestations religieuses. (Moise 2008: 2)

\section{Objectifs de la mission et contexte théologique}

Selon Picot, la mission confiée par le tsar Alexis s'inscrivait dans une suite d'efforts de la Russie "pour nouer des relations suivies avec les peuples de l'Asie Centrale et de l'Extrême Orient». Plusieurs émissaires avaient précédé Milescu, sans résultat. En 1669, Pazukin avait été envoyé à Khiva. En 1670, Daniel Arsinski «accompagné d'Ignace Milovanov, Antoine Filev, Grégoire Kobjanov» s'était mis en route vers l'Empire du Milieu. En 1674, le boïar Ivan Porsennikov «et deux négociants: Eustathe Filatjev et Gabriel Romanov ont repris sur son ordre le chemin de la Chine». La mission de Milescu était double. D'une part, il devait se rendre auprès de Gantimur, prince toungouse converti au

2 Spatar/Spathaire. Le titre de noblesse mineur de Nicolae Milescu signifie: Porte épée du prince régnant.

3 Section des sciences historiques, philologie et littérature, Institut d'études Sud-est européennes de l'Académie roumaine, Bucarest. 
christianisme, fixé en Sibérie. «La Chine ne cessait de réclamer ce personnage, qu'elle considérait comme rebelle. Il s'agissait de le fortifier dans ses bonnes dispositions [sa conversion à la foi chrétienne] et l'assurer que la protection du tsar ne lui ferait pas défaut.» Des questions de protocole - impliquant problèmes astrologiques, discussion du calendrier et évaluation du rang (divin? égal?) des dirigeants de deux empires - devaient être résolues par l'ambassadeur chargé d'établir «les titres que les souverains devaient se donner mutuellement» (Picot 1883 : 21). De même, il arrêterait «la langue à employer pour la correspondance entre la Russie et la Chine» (Picot 1883:21) avant de s'occuper de la rédemption de prisonniers et de la nature de leur échange. La seconde partie de la mission, géographique, incluait peut-être des fins économiques ou stratégiques. "Spatar devrait étudier les cours d'eau reliant la Sibérie à l'Empire chinois» (Picot 1883: 21, 22). L'historien Nicolae Iorga note dans sa préface de 1929 à des écrits inédits de Milescu un trait de tempérament littéraire: le Spathaire a été l'auteur d'écrits orthodoxes enflammés. Il a rédigé

une longue dissertation éloquente, injuste, injurieuse, évoquant les dangers amenés par «l'impie» Mahomet, avant de s'en prendre avec toutes les ressources de son intelligence et la fureur de son tempérament au Pape et à son «hérésie», qui lui paraît la pire de toutes, la plus dangereuse pour le vrai christianisme dont il se présente comme le défenseur. (Iorga 1929: 4)

Enfin, l'ouvrage qui a fait connaître Milescu dans le monde savant/religieux occidental de son temps, publié à Paris en 1669, est mentionné par Iorga comme une «contribution aux débats entre l'Église catholique et les calvinistes» (Iorga 1929: 2). Le livre a été donné à Stockholm à l'ambassadeur de France en Suède, Arnauld de Pomponne. Il s'agit d'«un opuscule, rédigé en grec et latin, qui parut, sous ce dernier vêtement, dans la réfutation des derniers par Armand et Nicole» (Iorga 1929: 2). Réédité en traduction roumaine aujourd'hui, cet écrit est présenté sur le web par son éditeur comme la preuve apportée des connaissances théologiques de son auteur. Par cet "Enchiridion, sive, Stella Orientalis Occidentalis splendens» rédigé en 1667, sur le modèle d'Érasme («Enchiridion militis christianis»), le Spathaire devient le troisième Roumain à "défendre avec ferveur l'orthodoxie face à l'offensive protestante». Cette «Étoile d'Orient brillant en Occident ${ }^{4}$ » est le premier écrit d' "un Roumain imprimé en Occident et la seule œuvre de Nicolae Spatarul Milescu imprimée de son vivant» (Diaconescu 1997: 1).

\section{Les trois versions et leurs translateurs}

a) L'importance et la nécessité de la version roumaine de Gheorghe Sion sont peut-être éclairées par une observation de Picot sur la faible diffusion des écrits russes du temps.

4 Traduction proposée (H. Lenz) pour «Steaua Orientului stralucind Occidentului». 
Tous les ouvrages composés par Spatar en Russie restèrent manuscrits. L'instruction était alors si peu répandue chez les Moscovites que l'imprimerie y était presque exclusivement employée pour les livres liturgiques. Des copies à la main suffisaient à la petite classe des lecteurs appréciant une œuvre littéraire. (Picot 1883: 20)

Préjugé ou réalité, il semble que Sion a traduit l'écrit de Milescu du grec en roumain, d'après une version établie par Chrysanthe Notaras «alors jeune clerc, futur élève des Universités de Padoue et de Paris puis patriarche de Jérusalem» neveu de Dosithée («grand patriarche de la Ville Sainte»). Si cet interprète a «employé aussi d'autres sources pour rendre en grec le contenu des mémoires et compilations de Milescu sur l'Extrême-Orient, on peut affirmer que le travail est dû presque en entier au Spathaire», considère N. Iorga. La preuve de paternité réside dans l'originalité des remarques linguistiques, historiques introduites dans ce récit de voyage chinois. On y a «noté avec orgueil que le roumain conserve des éléments du latin", on y mêle "l'histoire de la Chine à celle de Décébal le Dace», on y explique que la Dacie romaine coïncide avec les territoires de "Valachie, Bogdanie (Moldavie), Transylvanie» soit l'étendue de la Roumanie ultérieurement reconnue par le Traité de Versailles de 1919. On y évoque "Trajana ulpia» pour en donner le nom hongrois "Varhely». La rivière de Sargetia est le Streiu, dont le nom apparaît sous une forme roumaine [dans le texte grec]. Enfin, Iorga confirme que Milescu savait le grec et rédigeait dans cette langue, ce qui a pu faire de Notaras un simple transcripteur (Iorga 1929: 5). La biographie de Gh. Sion ${ }^{5}$ signale qu'il est né en 1822 à Mamornița (aujourd'hui en Ukraine), mort en 1892 à Bucarest. Il était issu d'une famille noble de Hârsova, județul / district de Văslui. Écrivain de Bucovine, il est devenu en 1868 membre titulaire de la récente Académie roumaine. En 1848, il avait pris part au mouvement révolutionnaire de Moldavie. Outre une œuvre personnelle (mémoires, pièces de théâtre, vers), il a traduit des classiques et romantiques français (dont Racine). Sa Descrierea Chinei/Description de la Chine date de 1888. Sa médiation a sans doute autorisé la reconnaissance de Milescu comme auteur patrimonial.

b) La version «en roumain, avec une préface» de E.M. Grigoraș, parue à Bucarest en 1926 dans la collection «Geografia românească » (XVII ${ }^{\mathrm{e}}$ siècle), est l'œuvre d'un auteur dont nous n'avons pas découvert d'autre trace. Conférencier, archiviste inconnu ou célèbre, il se présente comme un découvreur, déjà auteur d'un livre paru l'année précédente sur «Petre Stamiadi, philosophe du $\mathrm{XVIII}^{\mathrm{e}}$ siècle, dont le livre a été imprimé au Monastère de Neamt, en 1791. De cet auteur et de son œuvre, personne ne savait rien». Le Spathaire Milescu est aussi un «inconnu». En effet, si «ses aventures sont écrites et réécrites par tout le monde depuis Neculce, ses œuvres ne sont connues en Roumanie que

5 Voir l'article Gheorghe Sion en roumain https://ro.wikipedia.org/wiki/Gheorghe Sion, ou en anglais https://en.wikipedia.org/wiki/Gheorghe_Sion, pages consultées le 13 février 2015. 
de nom.» Sans nommer G.Sion ni C. Notaras, sans mentionner de date, le préfacier/traducteur fait état d'un fragment de Milescu déjà mis au jour. "Le "Journal sibérien" seul, et encore, traduit d'après une malheureuse traduction grecque» (Grigoras 1926: 3). En conséquence, il se propose de fournir dans son texte de 234 pages (source de la traduction ou de la langue du manuscrit traduit non précisée) une version complète du récit originel du Spathaire. Elle se composera de deux parties: "Jurnalul Siberian/Le Journal sibérien" et «Stateini Spisok/titre russe noté en caractères latins». Le sommaire divise de fait l'ouvrage ainsi:

- Préface, p. 3, - Carte [établie par Milescu sur son périple de Moscou à Pékin via la Sibérie, désignée en page 13, comme «Harta lui Milescu din cancelăria Moscovei / Carte de Milescu, de la Chancellerie de Moscou»], p. 13.

- Chapitre I - De Tobolsk à la Frontière de la Chine, p. 15 [titre et sous-titre en p. 15 «De Tobolsk à la frontière (Journal sibérien), date de départ: 15 avril $1675 »]$.

- Chapitre II - De la Frontière de la Chine au cours d'eau Năun, p. 48 [titre et sous-titre en p. 48: De la Frontière chinoise au cours d'eau Năun (Stateini Spisok), sans date].

- Chapitre III - Du cours d'eau Năun à Péking, p. 106. [idem en p. 106, sans mention de date sous le titre/sous-titre de chapitre mais le texte commence par «Le même jour (17 avril 1676)»].

- Chapitre IV - Le séjour à Péking, p. 136 [idem en p. 136, sans mention de date].

- Chapitre V - Le retour, p. 231 [idem en p. 231, sans mention de date sinon en corps de texte "Par conséquent, l'après-midi du $4 \ldots$ »]. Le récit de Milescu est achevé en p. 234 sur un paragraphe daté du 10 juillet.

L'écrit de Milescu est présenté comme d'importance mondiale. Sa valeur géographique est extrême dans la mesure où le parcours est celui d'un explorateur absolu, à l'origine d'une voie ferroviaire parmi les plus longues du monde. Il a tracé «la première route sèche entre l'Europe et la Chine. Avec peu de modifications, son trajet est suivi aujourd'hui par le Transibérien» (Grigoraș 1926: 4). Liant la géographie à l'esprit des relations internationales, Grigoraș considère que la «Diplomatie» possède dans ce livre un traité rare, montrant «Byzance face aux Peaux Jaunes». Car le récit est une dénonciation. "C’était la dernière occasion de témoigner au monde du côté inconnu de méthodes de gouvernement mises en lumière par Machiavel. Tout le livre, la partie IV surtout [le séjour à Pékin] est dans ce domaine un monument»(Grigoraș 1926: 5). L'importance historique du texte est majeure aussi. Elle marque un tournant prolongé au $\mathrm{xx}^{\mathrm{e}}$ siècle. L'écrit est témoin de «l'expansion russe en ExtrêmeOrient. Cette expansion confrontera ensuite la Russie et l'Angleterre, donnant naissance à la politique prête à dominer l'Europe des dernières décennies et qui sera la cause des derniers événements mondiaux» (Grigoraș 1926: 5). Les premiers inspirateurs d'une telle politique sont les Phanariotes [Grecs de 
l'Empire Ottoman administrant à Constantinople les Principautés roumaines] dont Milescu juge l'attitude. Grigoraș mentionne que la politique phanariote en Russie est antérieure à Pierre le Grand. Il pointe du doigt des «pogroms pratiqués sur les populations éloignées d'Asie " pouvant découler de cette politique et donc concerner par leurs répercussions une actualité roumaine d'après le Traité de Versailles de 1919. L'importance littéraire de Milescu enfin, est démontrée. Le Spathare a écrit et maîtrisé trois langues de culture de son temps: le slavon, le grec, le latin. Son excellence en matière de style et d'expression est donc fondée. «La clarté et la simplicité de son exposé d’un matériel scientifique vaste et complexe sont remarquables» (Grigoraș 1926: 6). La qualité esthétique, l'attractivité des descriptions de Milescu est affirmée. Le Spathaire décrit tantôt un temple aux inscriptions indéchiffrables, tantôt, en Chine du Nord, un temple avec une grande idole, des peintures de batailles etc. Il s'attarde sur un typhon à Pékin. Il évoque les religions multiples de la Chine, un Ministère de l'Astrologie, les superstitions des Chinois (Grigoraș 1926: 11). En somme, il se comporte en auteur comparable à Dimitrie Cantemir, prince moldave, premier polyglotte et polygraphe roumain, son «contemporain de réputation mondiale», reconnu par l'Académie de Berlin et en Angleterre (Grigoraș 1926: 12). On observe pour finir que la "Casa Școalelor» publiant cette enthousiaste réécriture de Milescu a fonctionné, selon le web, de 1896 à 1948. Elle avait été fondée par décret-loi («Lege») le 20 janvier 1883, c'est-à-dire l'année de la publication à Paris du mémoire de Picot, auquel se réfère le début de l'exposé.

c) La version de Corneliu Bărbulescu (1974), est l'œuvre d'un chercheur de l'Institut de folklore et ethnographie de Bucarest, spécialiste de l'étude du conte roumain et un de ses promoteurs majeurs au XIX ${ }^{e}$ siècle: Petre Ispirescu. Sa réécriture procède donc d'une compétence scientifique reconnue. Elle a été réalisée pour une collection de vulgarisation des classiques nationaux (Biblioteca Eminescu, Editura Eminescu). La quatrième de couverture signale les noms d'auteurs suivants, publiés l'année précédente (1973), écrivains du début du Xx ${ }^{\mathrm{e}}$ ou prédécesseurs du XIX ${ }^{\mathrm{e}}$, tous reconnus comme représentatifs de la littérature nationale: le premier est Tudor Vianu (théoricien littéraire, stylisticien), suivi de Mihail Sadoveanu (romancier moldave, académicien, auteur de plus de cent livres), Liviu Rebreanu (romancier transylvain de l'entre-deux-guerres faisant état de tensions entre Roumains et Hongrois), Zaharia Stancu (romancier fécond promu par le communisme, dans la lignée de Gorki, avec «Desculț / Pieds nus»), Ionel Teodoreanu (romancier moldave de l'enfance enchantée), B.P.Hașdeu (théoricien culturel de $\mathrm{XIX}^{\mathrm{e}}$, connaisseur de littérature roumaine ancienne), Ion Creangă (classique fondateur de la langue roumaine au XIX ${ }^{e}$, avec Eminescu et Caragiale), enfin Călinescu, théoricien et romancier du milieu du $\mathrm{xx}^{\mathrm{e}}$, publié avec une Vie d'Eminescu, le poète classique national du XIX ${ }^{\mathrm{e}}$ dont la valorisation bat son plein à l'époque ceaușiste. L'avant-propos de C. Bărbulescu détaille les étapes biographiques ayant permis «la notoriété européenne» du lettré moldave. Dès 1664, fuyant Constantinople en raison de l'échec politique de Grigore Ghica, prince de Muntenie, le Spathaire apparaît à la cour de Frederich 
Wilhelm comme un grand savant, connaisseur de langues de grande circulation. L'ambassadeur de France en Suède le recommande pour sa compétence aux jansénistes de Port Royal en 1666 et Louis XIV mentionne s'être entretenu avec lui. En 1669, son nom est connu à Paris, pour la publication de son «Enchiridion». Cité par le pasteur calviniste Claude en 1671, il est recommandé par le Métropolite Dosithée de Jérusalem au tsar Alexei Mihailovici à Moscou, en qualité de polyglotte instruit. La "gloire» de Milescu est augmentée suite à son retour de Chine, où il est envoyé «en qualité d'ambassadeur auprès de l'empereur Kang-Hi». C. Bărbulescu cite les nombreuses sources et savants de référence ayant eu connaissance d'une version du Voyage en Chine. Le linguiste suédois Sparwenfeld, résidant à Moscou entre 1684 et 1687 y a rencontré le Spathaire qui lui a offert en 1685 un manuscrit de sa Description de la Chine aujourd'hui conservée à Paris (Bibliothèque nationale). En 1692, le Hollandais, N. Witsen rapporte dans son livre sur la Tartarie, paru à Amsterdam, que le jésuite Grimaldi a admiré à Pékin l'exceptionnelle culture du Spathaire lors de sa rencontre avec l'empereur Kang $\mathrm{Hi}$, dont il a été témoin. Leibniz en personne aurait interrogé Witsen sur Milescu, auteur de «l'Enchiridion... », en 1699. De ces échos, C. Bărbulescu conclut que les «écrits géographiques et ethnographiques » de Milescu ont dû avoir une audience considérable dans la science de l'époque. Nombre de ses observations sont parvenues à la connaissance d'auteurs divers pour «enrichir les données documentaires du temps puisque la "Description de la Chine" a circulé en nombreuses copies manuscrites, avant le début de l'impression de l'œuvre de Milescu en 1882» (Bărbulescu 1974: VII; - nature, langue, lieu de l'imprimé non précisés). Surtout, la fin de la préface insiste sur un trait ignoré des prédécesseurs. Milescu «peut être vu aussi comme le fondateur de l'ethnographie russe». Écrivain géographique, il a effectué «la première description connue du lac Baïkal, découvert par les Russes dans la première moitié du XVII ${ }^{\mathrm{e}}$ siècle». Il a enregistré des données nouvelles sur la Sibérie, tout en "corrigeant des erreurs de prédécesseurs dont il amende les résultats». Sa description du "peuple ostiaque est le premier document ethnographique russe mentionnant ce dernier» outre qu'il constitue un morceau d'anthologie (Bărbulescu 1974: VIII, XIX). Enfin, la deuxième partie du livre, «le Document d'État», diffère du récit du voyage en Sibérie par son descriptif de la mission diplomatique proprement dite en termes sociologiques voire psychologiques. La préface l'évoque de manière succincte. On y parle «des hommes, des relations avec les Chinois, des conversations et traités contractés, d'affaires diplomatiques». La manière de Milescu est évoquée en termes abstraits mais élogieux: maîtrise et intelligence de la gradation du récit, esprit pénétrant, description détaillée et colorée du protocole, appréciations suggestives, émotion face à la beauté des monuments, charme de l'écriture. Le plan de l'ouvrage de Bărbulescu, divisé en deux, est le même que celui de Grigoras quoique sa version soit plus longue (330 p.), en raison du texte-source sans doute. Une note sur l'édition précise en tout cas que la langue source de la traduction est le russe (première publication à Saint Petersbourg), que l'édition de travail est 
soviétique. Quant à ses visées, elles sont caractéristiques d'une vision du savoir liée à une politique.

Cette édition est la première traduction roumaine intégrale du JOURNAL publié par Arseniev (en note 1 et en caractères latins: Kniga i v nei pisano putesestvie țarstva Sibirskogo ot goroda Tobolska i do samogo rubeja gosudarstva rubeja gosudarstva Kitaiskogo, St Petersburg, 1882: Stateini spisok psolstva v Kitai N.Spafaria, St-Petersburg, 1906) revue d'après les textes publiés par l'Académie des Sciences de l'URSS, dont le contenu est équivalent. Nous avons rendu la terminologie spécialisée en transcription phonétique et orthographe courante, en cas de nécessité nous avons ajouté des explications en note de bas de page. En général, notre traduction s'est efforcée de conserver «l'atmosphère» stylistique de l'original pour éviter d'altérer les attributs de l'époque où il a été écrit. Nous avons tenté de proposer aux lecteurs contemporains un document "authentique " du XvII siècle, susceptible d'être utilisé - par ceux qui le veulent - comme texte de référence pour la recherche scientifique, ou à des fins didactiques. C.B (Bărbulescu 1974: XI)

Qu'entendre par réécriture? L'exercice peut inclure le recopiage d'un alphabet dans un autre (et les erreurs/divergences d'interprétation du texte qui en découlent), la notation de l'oralité, le résumé ou, à l'inverse, l'extension d'un écrit par glose intégrée, développement explicatif. Il peut équivaloir à une traduction/translitération, cette dernière pouvant se prolonger dans la translation au sens de Berman, soit dans une traduction intégrant la présentation au plus juste d'un auteur, méconnu ou calomnié telles les réécritures de Milescu par Sion, Grigoraș, Bărbulescu vues plus haut. Toutes ces modalités de notation ont fait partie de l'expérience de la langue/culture roumaine, des débuts à nos jours. Au milieu du XIX ${ }^{\mathrm{e}}$ siècle encore, le roumain, d'origine latine, était noté en caractères cyrilliques. Les trois versions examinées du texte du Voyage en Chine, ont souhaité prolonger, avec un parti pris quasi-hagiographique, le récit d'un Milescu vu comme un incompris en Occident, en dépit de sa reconnaissance en France, déclencheur possible de sa valorisation tardive dans son propre pays. On se souviendra ainsi que le Voyage en Chine a été montré au cours de son histoire de manuscrit par le Britannique J.F. Baddeley comme un plagiat mot à mot de l'Atlas de la Chine du Jésuite Martinius Martini (Bărbulescu 1974: VI, note 1). Il a été aussi soupçonné d'être l'œuvre d'un aristocrate brigand et d'un faussaire.

Les Roumains n'étant pas connus comme voyageurs, explorateurs, navigateurs de grands espaces, ce dénigrement de Milescu, inséparable de fabulations biographiques qui ont longtemps prévalu sur l'établissement de son texte, a son importance. Avant le $\mathrm{xx}^{\mathrm{e}}$ siècle, on se ridiculiserait à comparer un voyageur roumain aux explorateurs italiens, espagnols, portugais, découvreurs de continents, aux Catalans sillonnant l'espace méditerranéen. À l'inverse d'autres locuteurs de langues romanes riverains de l'Océan, des mers Méditerranée, Adriatique, Égée et au-delà du Pont-Euxin (cette mer fermée, ouverte aux Vénitiens avant le $\mathrm{xv}^{\text {e }}$ siècle, transformée en «lac turc», sous domination 
ottomane, car la navigation italienne n'y était plus permise), les ressortissants de Principautés n'ont eu accès qu'à des mers nordiques, à la mer Noire surtout et encore pour une petite part d'une Moldavie disputée aux voisins. Il est donc plausible que la seule réalité du voyage de Milescu ait été mise en doute, que son apport scientifique à une connaissance «européenne» de la Chine ait été minoré sinon nié car ignoré. Les réécritures du récit de son périple en diverses langues du monde chrétien orthodoxe, au lieu de se voir considérées comme la première condition de transmission de son message, n'ont dû faire qu'obscurcir la preuve de la réalité observante de ce dernier, faisant régresser le statut d'un récit d'auteur relevant de la "sinologie scientifique» représentée par les Hollandais selon Charles le Blanc (XVIII ${ }^{e}$, XIX ${ }^{e}$ siècles), voire de la "sinologie religieuse" illustrée en Occident par des missionnaires catholiques surtout jésuites (XvI et XVII ${ }^{\mathrm{e}}$ siècles) jusqu'au stade inaugural de la «sinologie imaginaire» dont Marco Polo est le représentant sans rival sans doute mais dépassé.

\section{Bibliographie}

Bărbulescu / Spătarul Milescu N., 1974, Jurnal de călătorie în China, traducere, cuvînt înainte, indicați bibliografice de Corneliu Bărbulescu, București, Editura Eminescu.

Diaconescu T., 1997, résumé sur l'ouvrage Manualul sau Steaua Orientului stralucind Occidentului [Enchiridion], Milescu Spàtarul Nicolae, publié dans la collection «Romania Orientalis», Studii clasice, avec une préface de Traian Diaconescu, 160 p., http://www.euroinst.ro/.

Grigoraș/Spătarul Milescu N., 1926, [în românește și cu o prefață de Em.C. Grigoraș], Călătorie în China - 675-1677, cu o hartă-, Geografia românească, sec. XVII, București, Editura Casei Scoalelor.

Iorga N., 1929, Euvres inédites de Nicolas Milescu publiées par N. Iorga, Bucarest, Ateliers graphiques Cultura Națională, Académie roumaine, Études et recherches III, Bucarest.

Le Blanc C., 2007, Profession sinologue: les trois phases de la sinologie, Presses de l'Université de Montréal, nouvelle édition: http://books.openedition.org/ pum/497.

Picot É./Milescu Nic. S., 1883, Notice Biographique et Bibliographique sur Nic. Spatar Milescu, Ambassadeur du Tsar Alexis Mihajlovic en Chine, Paris, Ernest Leroux éditeur, Librairie de la société Asiatique de l'École des langues orientales vivantes.

Solunca Moise E., 2008, «Spătarul Nicolae Milescu (1636-1708)», Ziarul Curentul, 10 octombrie 2008, https://www.curentul.info/cultura/spatarulnicolae-milescu-1636-1708. 
Surcel V., 2013, «Nicolae Milescu Spătarul, cărturarul român care a spionat China imperială», Ziarul Curentul, 15 iulie 2013, https://www.curentul.info/special/ nicolae-milescu-spătarul-cărturarul-roman-care-s-spionat-China-imperială/. 